\title{
UV photochemical synthesis of heparin-coated gold nanoparticles
}

\author{
M. del P. Rodríguez-Torres • Luis Armando Díaz-Torres • \\ Pedro Salas • Claramaría Rodríguez-González • \\ Martin Olmos-López
}

Published online: 24 September 2013

(C) The Author(s) 2013. This article is published with open access at SpringerLink.com

\begin{abstract}
Sodium salt heparins of both reactive and pharmaceutical grade were used as the reducing and stabilizing agents for the UV photochemical synthesis of gold nanoparticles. Reduction of chloroauric acid, $\mathrm{HAuCl}_{4}$, by heparin using UV irradiation as a catalyst is a simple, inexpensive method with direct biofunctionalization of the products for further use. Experiments using both heparin types were carried out separately. The varying synthesis parameters were the $\mathrm{HAuCl}_{4}$ and heparin sodium concentrations. The synthesized AuNPs present spherical as well as anisotropic shapes, such as oval, triangular, hexagonal sheets, rods, and some other faceted forms, with dimensions ranging from 20 to $300 \mathrm{~nm}$ as well as agglomerated networks. No usage of additional surfactants or growth-inducing seeds was necessary in the syntheses.
\end{abstract}

Keywords Gold colloids · Photochemistry · Green synthesis · Glycosaminoglycans $\cdot$ Spectroscopy

\section{Introduction}

Colloidal metal nanoparticle synthesis techniques are used to produce different types of nanoparticle shapes for several applications, such as drug carriers, tumor detectors, photothermal agents, and so on. Chemical methods include chemical reduction of metal salts [1-3], alcohol reduction and polyol processes

\footnotetext{
M. del P. Rodríguez-Torres · L. A. Díaz-Torres $(\bowtie)$

M. Olmos-López

Grupo de Espectroscopia de Materiales Avanzados y

Nanoestructurados (GEMANA), Centro de Investigaciones en

Optica A. C., León Gto. 37150, Mexico

e-mail: ditlacio@cio.mx

P. Salas · C. Rodríguez-González

Centro de Física Aplicada y Tecnología Avanzada, Universidad Nacional Autónoma de México, A.P. 1-1010, Querétaro 76000, Mexico
}

$[4,5]$, microemulsions [6], thermal decomposition [7], and electrochemical synthesis [8]. Physical methods include the exploding wire technique [9], plasma synthesis [10], chemical vapor deposition [11], microwave irradiation [12], supercritical fluids [13], sonochemical reduction [14], and gamma irradiation [15]. Photochemical synthesis is now being considered as another alternative for obtaining colloidal nanoparticles [16]. Some of its advantages are high spatial resolution, reaction selectivity and controllability as well as shape conversion. This method can be used with light sources like lasers [17], medium and low pressure Mercury [18], Xenon, LEDs and UV lamps [19], and even white light [20].

A wide range of materials and their combinations are available for light-based synthesis in solution; metal salts which act as precursors $\left(\mathrm{AgNO}_{3}, \mathrm{HAuCl}_{4}\right.$, and $\left.\mathrm{PtCl}_{6}\right)$ in a suitable solvent (ethyleneglycol, alcohols); chelate complexes, metal acetylides, polymers, and surfactants. There are also photosensitive agents that assist the reactions by means of radical formation which allow the metal reduction process leading to nanoparticle generation [17].

Heparin (also known as unfractioned heparin) is a member of the glycosaminoglycans family of carbohydrates and has a complex and heterogeneous structure. Its molecular weight can vary from 5,000 to 40,000 Da; and its sulfation degree also varies depending on the tissue it is collected from, which can be either beef lung or porcine intestinal mucosa, with $75 \%$ of heparins made from the latter. It is formed mostly by two repeating disaccharide units (variably sulfated) shown in Fig. 1, a 2-O-sulfated iduronic acid (left ring) and a 6-Osulfated, $\mathrm{N}$-sulfated glucosamine (right ring) which can also be referred to as only iduronic acid and glucosamine, respectively. Due to the sulfate groups present, this disaccharide structure makes heparin the highest negative chargecontaining of any known molecule.

Heparin is highly sensitive to light exposure (especially in the UV range), making it an excellent candidate for metal 


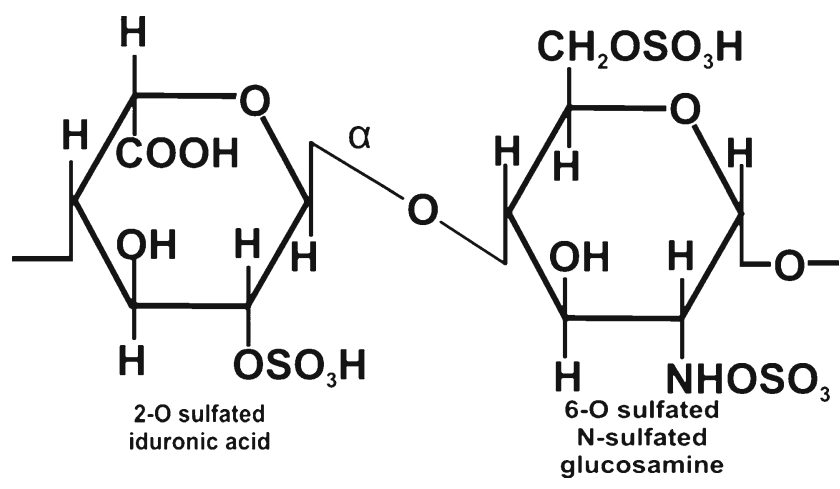

Fig. 1 Sodium heparin structure

nanoparticle synthesis. It is a very reactive material due to its composition; for example, each disaccharide repeating unit contains a carboxyl group. All repeating units contain one or more $1^{\circ}$ or $2^{\circ}$ hydroxyl groups and an average of 2-2.5 sulfo groups and N-sulfo groups are found in 75-85\% of all repeating units. Approximately $15-25 \%$ of these repeating units contain a vicinal diol. Each chain contains a reducing hemiacetal end. Heparin also contains $\sim 0.3$ unsubstituted amino groups/chains and it owes its acidic nature to the covalently linked sulfate and carboxylic acid groups [21].

In this work, a photochemical method involving UV irradiation and the usage of sodium heparin, well-known biomolecule as a reductant and stabilizer in a green synthesis scheme has been developed in order to get nanowire-like, anisotropic and plate-shaped nanoparticles at room temperature. Green synthesis methods are good alternatives for avoiding the usage and generation of hazardous materials [22-24]. These forms have already been synthesized by heat-based techniques [25] at low and ambient temperatures [26] and via photochemical methods [27] not using heparin as a reagent whereas UV triggering has been employed. Heparin has been used in thermal-based methods obtaining spherical nanoparticles [28-31].

The samples obtained were characterized by UV-vis spectroscopy and TEM; the concentration of $\mathrm{HAuCl}_{4}$ and heparin was varied in order to find out how this could affect the nanoparticle shape and plasmon peak. Also, heparin changes before and after UV irradiation were studied by means of UVvis, FTIR spectroscopy and $\mathrm{pH}$ measurements. Finally, a formation mechanism was investigated and proposed.

\section{Experimental}

Materials

Gold (III) chloride trihydrate $99.9 \%$ metal basis $\left(\mathrm{HAuCl}_{4}\right)$ and reactive-grade heparin sodium salt (195.9 USP units/mg) were purchased from Sigma-Aldrich. Pharmaceutical-grade heparin in aqueous solution $(5,000 \mathrm{IU} / \mathrm{mL}, 10 \mathrm{~mL}$, sealed container) was obtained from PISA Laboratories. Deionized water was used for preparing stock solutions as well as a solvent in all synthesis reactions. The materials for the experimental procedures were used as received without any further purification or modification.

All glassware and quartz material was cleaned with aqua regia (three parts hydrochloric acid and one part nitric acid), then rinsed with distilled water and $96 \%$ ethanol. All washed material was dried in an oven at $70{ }^{\circ} \mathrm{C}$ before synthesis procedures take place. Gold (III) chloride trihydrate and reactivegrade heparin stock solutions were prepared using deionized water. Pharmaceutical-grade heparin was used as received.

UV irradiation of solutions

For nanoparticle synthesis, a home-made cylindrical UV reactor was used. Inside the reactor there are three vertically oriented black light lamps (peak intensity at $\lambda=366 \mathrm{~nm}, 4 \mathrm{~W}$ ). Forced air cooling through top of the reactor was used to keep a reaction temperature around $30{ }^{\circ} \mathrm{C}$. The typical synthesis solution is prepared as follows: First, deionized water is added, secondly the gold (III) chloride trihydrate, and finally the heparin, this solution is then poured in a $10-\mathrm{mm}$ quartz-cell which is placed inside the reactor in the central position for it to be irradiated uniformly for seven hours as shown in Fig. 2.

In order analyze UV effects on heparin, stock solutions of heparin alone were poured in a $10-\mathrm{mm}$ quartz cell and placed inside the UV reactor to be irradiated up to seven hours. The UV-vis and FTIR spectra as well as $\mathrm{pH}$ changes were continuously monitored.

Synthesis of gold nanoparticles with reactive-grade sodium salt heparin

Two sets of experiments were carried out, the first one keeping a constant concentration of heparin at $0.0312 \mathrm{mM}$ while

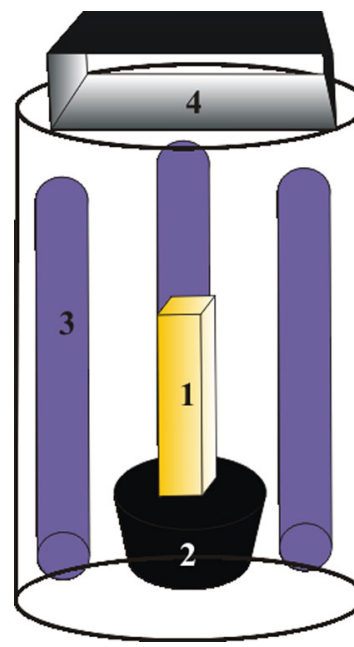

Irradiation time:7 hours

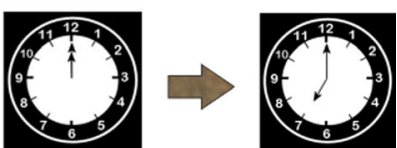

UV reactor:

1.Quarz cuvette.

2. Cuvette base.

3.UV-lamp.

4.Airing system.

Fig. 2 Block diagram of the UV reactor used for nanoparticle synthesis 
increasing the gold precursor concentration :0.0833 (sample A1), 0.333 (sample A2), and $0.4165 \mathrm{mM}$ (sample A3). The second consisted of maintaining a gold concentration of $0.833 \mathrm{mM}$ and varying the heparin one by $0.312,0.624$, $0.936,1.248$, and $1.56 \mathrm{mM}$.

Synthesis of gold nanoparticles with pharmaceutical-grade sodium salt heparin

The experiments carried out followed exactly the same preparation procedure for the reactive-heparin samples. Since pharmaceutical-grade heparin its vial has a $0.833-\mathrm{mM}$ concentration, a maximum of $0.7 \mathrm{mM}$ was used.

\section{Characterization}

UV-vis spectra were measured in a Perkin Elmer Lambda 3B $\mathrm{UV}-$ vis spectrophotometer with a 1-mm quartz cell; the measurements were done in air at ambient temperature (for both heparin solutions and colloidal nanoparticle samples). For infrared spectra measurement, a Perkin Elmer Spectrum BX FTIR system was used. Solid samples were prepared by mixing heparin powder with $\mathrm{KBr}$ (potassium bromide), in a heparin to $\mathrm{KBr}$ ratio of $1: 100$, and milling them to form a very fine and homogeneous powder to make a pellet for obtaining the spectra. Pharmaceutical-grade heparin, as well as both UVirradiated and non-irradiated heparin solutions, were dried at $45^{\circ} \mathrm{C}$ for $6 \mathrm{~h}$, and mixed with dry $\mathrm{KBr}$ powder. The $\mathrm{pH}$ changes were monitored by using a Conductronic $\mathrm{pH} 120 \mathrm{pH}$ meter. Transmission electron microscopy (TEM) was carried out in a JEOL model JEM-1010 operated at $80 \mathrm{kV}$ using a carbon grid. TEM samples were prepared in the traditional way; an aliquot of the solution was dropped on a $3 \mathrm{~mm}$ diameter lacey carbon copper grid and then left to dry at room temperature for the solvent to evaporate.

\section{Results and discussion}

\section{Reactive-grade heparin results}

The synthesized samples were prepared by varying the concentrations of both, HAuCl4 and heparin. First, the HAuCl4 concentration was increased from $0.0833 \mathrm{mM}$ up to $0.4165 \mathrm{mM}$,
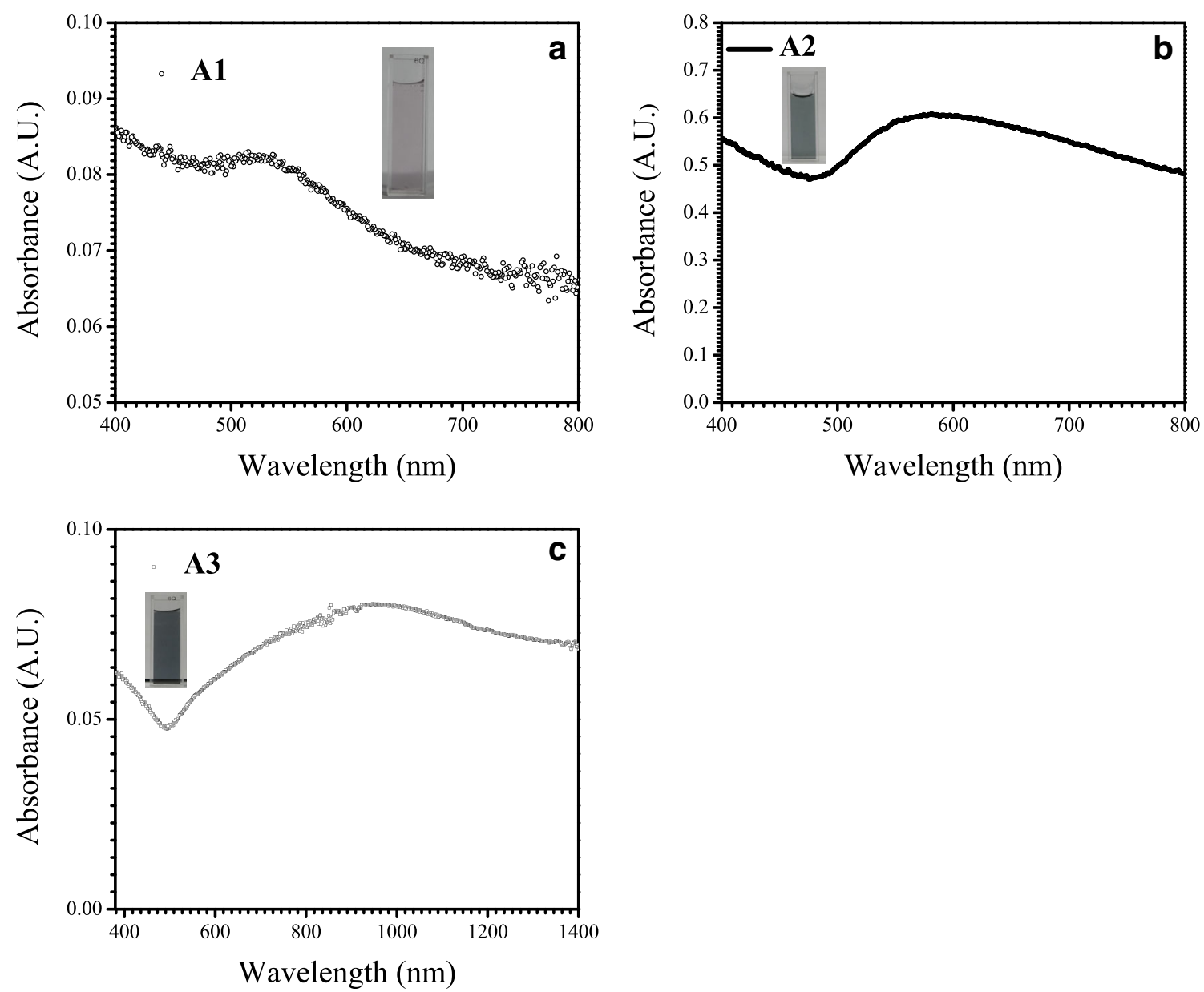

Fig. 3 a, b, c UV-vis spectra of the nanoparticles prepared with increasing gold concentration; the solution tones are also shown along 
with heparin fixed at $0.0312 \mathrm{mM}$, and irradiated up to seven hours. Figure 3 shows some UV-vis spectra of samples A1, $\mathrm{A} 2$, and $\mathrm{A} 3$, for increasing $\mathrm{HAuCl} 4$ concentrations of 0.0833 , 0.333 , and $0.4165 \mathrm{mM}$, respectively. The solutions color changed from a light purple to a blue-grayish hue, this suggests a change in the metal oxidation and the type of nanoparticles formed. Additionally, the UV-vis spectra also suggest particle shape changes.

For sample A1, Fig. 3a, the spectra shows a surface plasmon resonance (SPR) peak centered at $522 \mathrm{~nm}$, which is typical for nanospheres. The TEM micrographies in Fig. 4a, b show sphereroid particles of small diameters between 16 and $18 \mathrm{~nm}$. It is clear that the nanoparticle yield is quite low, being in agreement with the small amplitude of the SPR peak in Fig. 3a. Besides this, there are some aggregates in a wire-like assembly, although they are still attached to much bigger clusters. This is in accordance with its UV spectrum because the shape is quite wide indicating the dispersion provoked by the aggregated assemblies and the maximum absorbance value being quite low. Samples A2 and A3 differ from A1 in their color and optical density as shown in Fig. $3 \mathrm{~b}$ and c. These two

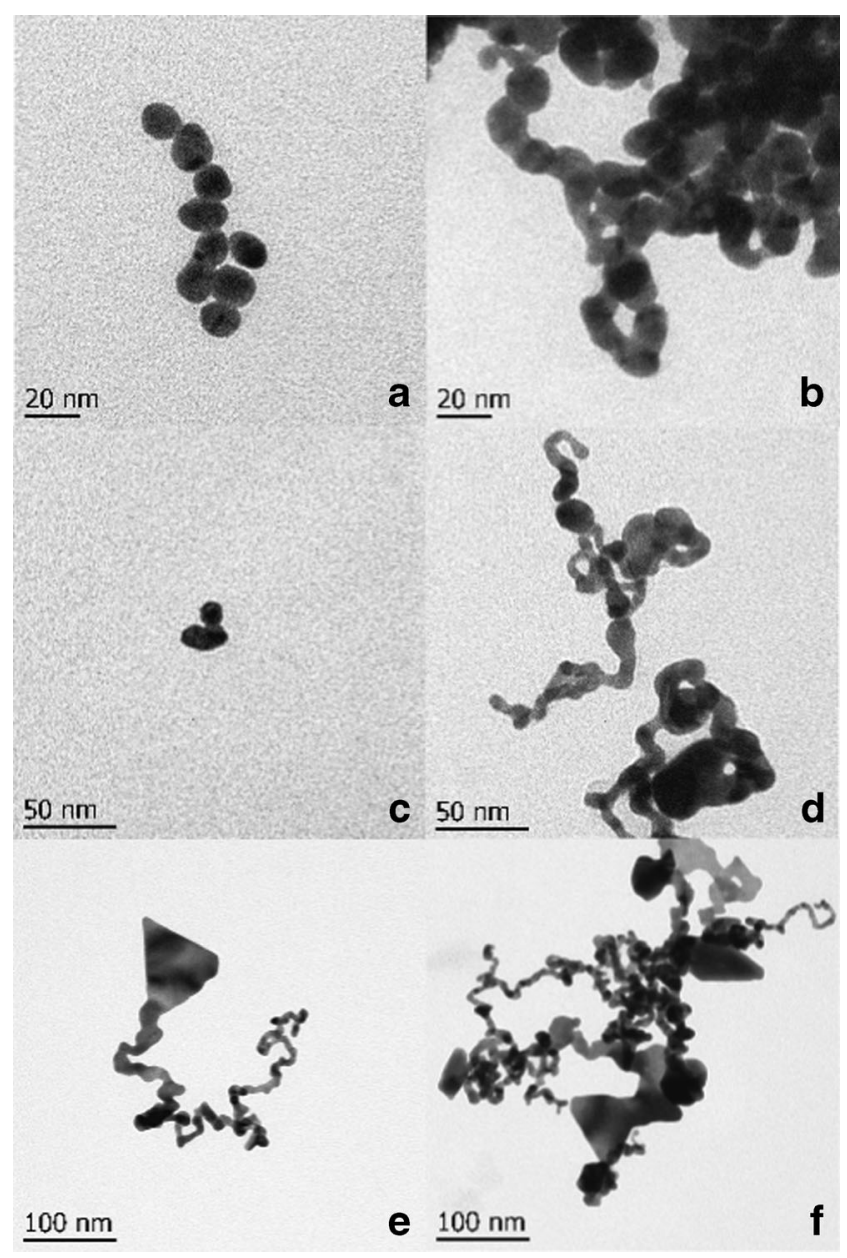

Fig. 4 TEM images of a, b sample A1, c, $\mathbf{d}$ sample A2 and e, $\mathbf{f}$ sample A3 solutions show similar tones being A3 the one with a more intense shade due to the higher gold concentration. Their plasmon peak is shifted to longer wavelengths respect to the A1 plasmon, also other weak peaks in the near IR could be observed.

Nanoparticles in A1 are spheroids, which are in agreement with their UV-vis spectrum, but it has to be taken into account that the distribution is broad probably because the particles tend to agglomerate a little and besides that they have different sizes. Sample A2 exhibits very few oval-like nanoparticles whereas there is a lot of wire-like particles that are less aggregated and more elongated, and still keeping some nanoparticles within, see Fig. 4c, d. In Fig. 4e, f, triangular plate-like nanoparticles are observed in addition to the nanowires. Sample A3 contains nanoparticles on the order of $100 \pm 50 \mathrm{~nm}$ as well as other quasispherical nanoparticles between 10 and $30 \mathrm{~nm}$, most of which are agglomerated. This can also explain why in its UV-vis spectrum a depression is found at $495 \mathrm{~nm}$.

A possible explanation for the change from spheres to nanowire networks could be the fact that when the metal precursor concentration is very low, nanoparticles are formed, but the lack of stabilizer tends to form aggregated, elongated and bigger-sized structures. Taking into account that the gold concentration was being increased, the preferential growth in some directions is reached. This could explain the changes in shapes and the increase in length and decrease in diameter, indicating that there is not enough capping agent to passivate the nanoparticles surface.

The particles that have a plate-like aspect like in sample A3 could be a sign of polycrystallinity because of the varying contrasts in the TEM images. This may suggest that the crystals are oriented in different directions as the electron beam passes through the sample. Vasilev et al. [32] found similar results but using 2-mercaptosuccinic acid (MSA) as the reducing and stabilizing material, obtaining similar wire diameters and plate dimensions, but shorter wires. Other research groups have also obtained comparable results [33, 34].

When the heparin concentration was increased, the synthesized samples show narrower SPR peaks around $532 \pm 4 \mathrm{~nm}$ that slightly shift towards longer wavelengths, show less dispersion, and increase in amplitude as heparin concentration increases, as can be observed in Fig. 5, the solutions color go from a light purple up to purplish-blue one, indicating nanoparticles' change in shape and size. When the highest heparin concentration is reached there is again a slight increase in dispersion, but still less than the one for the lowest heparin concentration.

This could be due to the fact that there are several assemblies formed by nanowires as well as some other nanoparticles of irregular shapes as shown in Fig. 6a. The high dispersion of the SPR at the lowest heparin concentration might be a consequence of the presence of several clusters formed by nanowires and some small nanoparticles within their structures; see 


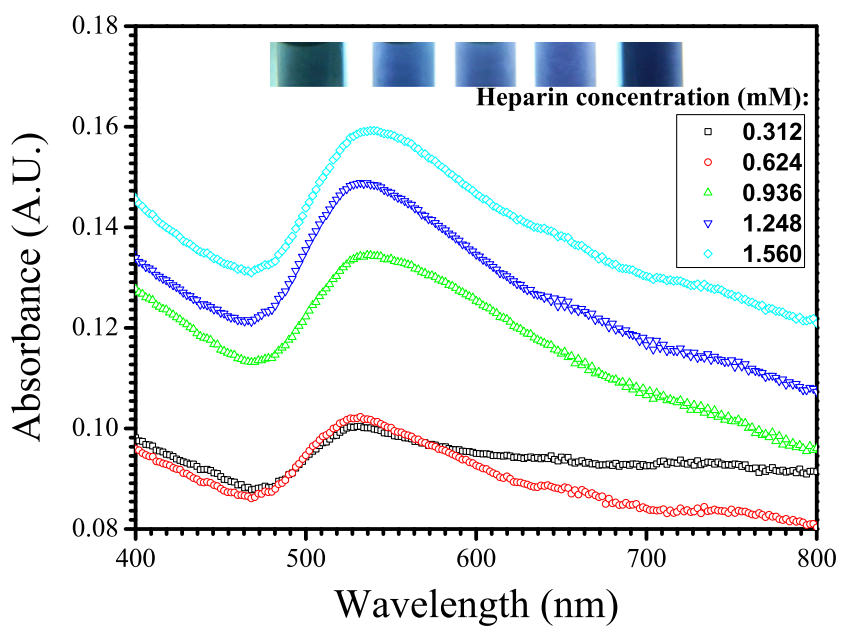

Fig. 5 UV-visible spectra of samples prepared with increasing heparin concentration. On top, there are the synthesis produced and their tone changes

Fig. 5. As the heparin concentration increases, the assemblies begin to connect to each other forming networks of different sizes. The reason for this may come from the fact that heparin

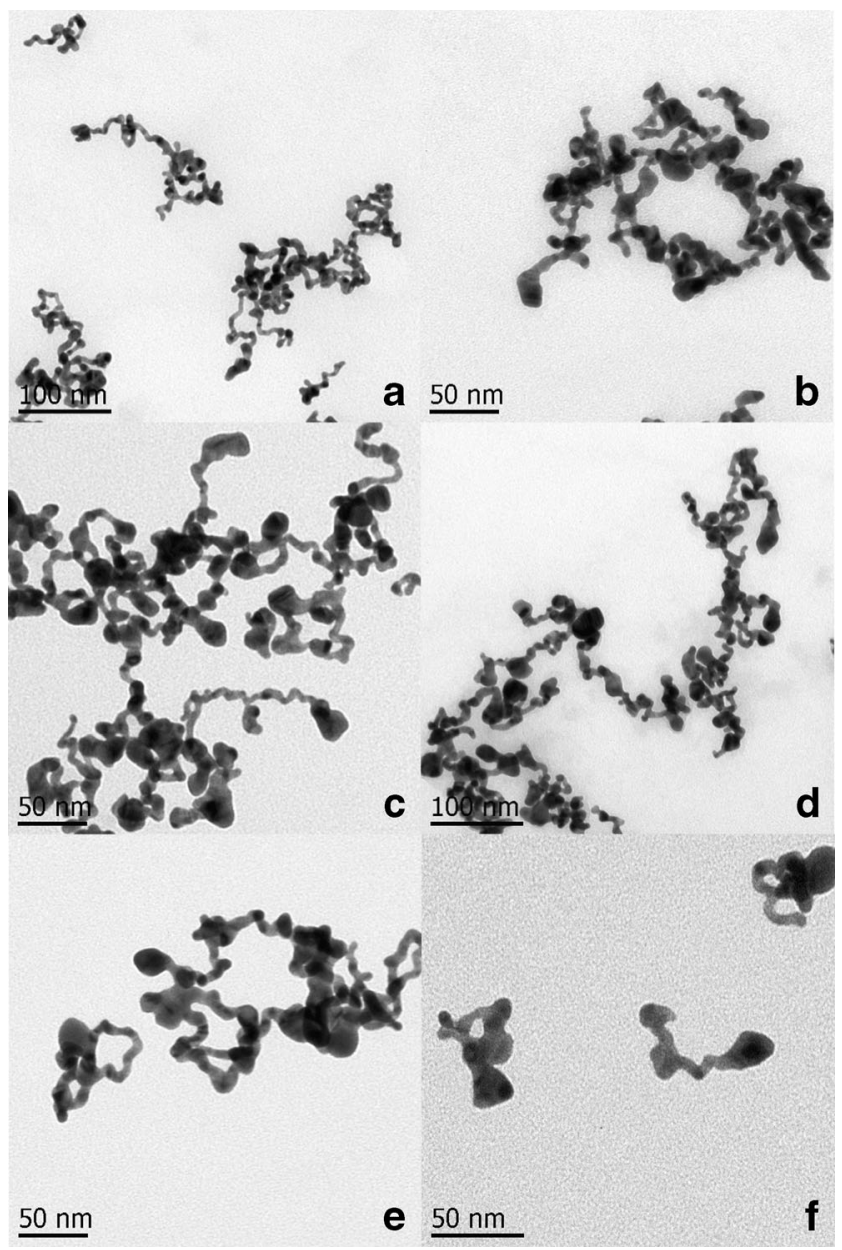

Fig. 6 TEM images when increasing heparin concentration. a 0.312 , b 0.624 , c 0.936 , d 1.248 and e, f $1.56 \mathrm{mM}$
HEP1

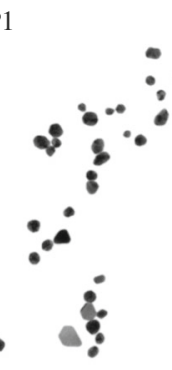

$200 \mathrm{~nm}$

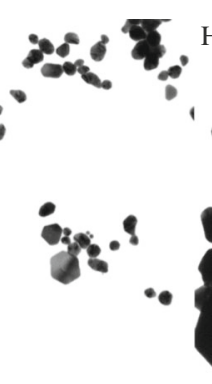

$200 \mathrm{~nm}$
Fig. 7 HEP1 and HEP2 samples TEM images

starts to enclose nanoparticles as well as to stabilize them, so this causes the individual clusters formed to gather in networks, and at the same time it induces growth for some nanoparticles in some preferential directions. Their aggregation inside the wires is probably a result of the heparin encapsulation, mentioned before, that is not letting the nanoparticles grow outside the wire but inside it; and in consequence inducing their aggregation in some parts. There is a noticeable difference at the highest concentration, there are still formed networks but now some parts of them break apart from that main structure, in a tap-pole shaped nanoparticles (see Fig. 6f). That could make a slight dispersion contribution to the SPR peak observed in the UV-vis spectrum in Fig. 5. Similar nanoparticles shapes and networks have been obtained in previous works by means of photochemical synthesis using a mix of CTAB, $\mathrm{NaOH}$ and deionized water to reduce $\mathrm{HAuCl} 4$ with $365 \mathrm{~nm}$ UV light for about $9 \mathrm{~h}$ at room temperature [34].

Pharmaceutical-grade heparin results

The concentrations used for increasing gold concentrations are 0.0833 and $0.4165 \mathrm{mM}$ (keeping heparin at $0.0312 \mathrm{mM}$ ) and for increasing heparin concentrations (keeping gold at $0.833 \mathrm{mM}$ ) are $0.312,0.5$, and $0.7 \mathrm{mM}$.

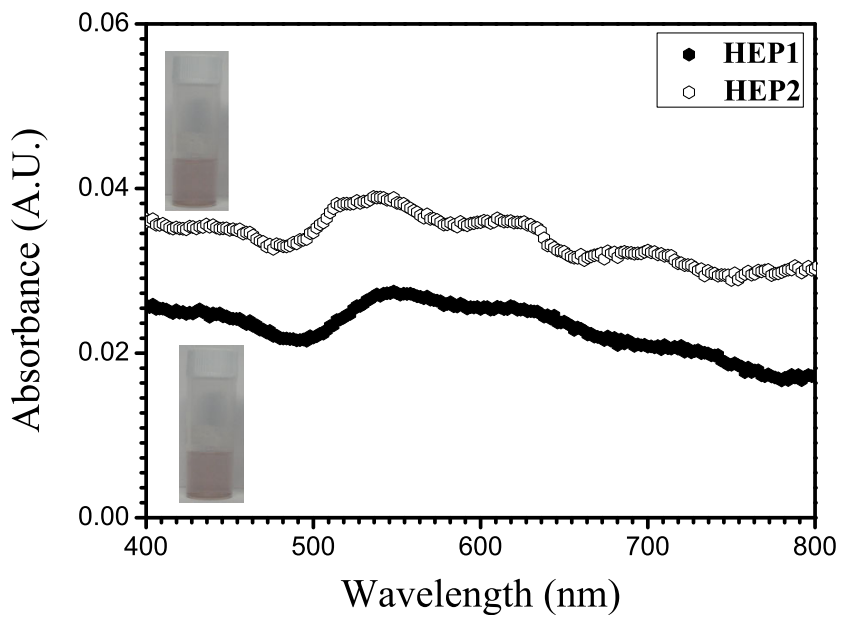

Fig. 8 UV-vis spectra of samples synthesized with decreasing gold concentration along with the prepared colloids 
Fig. 9 HEP3, HEP4 and HEP5 samples TEM images

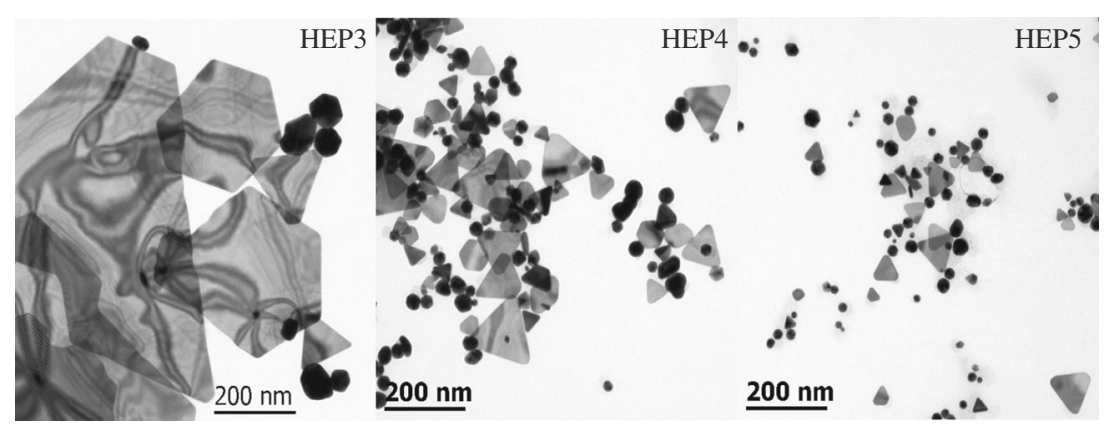

Non-spherical gold nanoparticles present multiple absorption bands correlating with their multiple axes, and they can support both propagating and localized surface plasmon resonances. The number of SPR peaks usually increases as the symmetry of nanoparticles decreases; spherical nanoparticles exhibit only one peak, whereas two and three peaks are often observed in nanorods, nanodisks, and triangular plates, respectively [30]. Nanoplates have been previously synthesized by photochemical methods [23,35-37] with similar results. In our case, when first modifying the gold concentration, it is observed that for the HEP1 sample, few nanoparticles are generated and they are small, ranging from 13 to $20 \mathrm{~nm}$. For sample HEP2 the situation is completely different because the nanoparticle sizes go up abruptly most likely due to the fact that the capping effect coming from heparin is not enough to stop their growth as shown in Fig. 7.

Also, another thing is that in contrast with reactive-grade heparin experiments, there is now a shape change. The particles show anisotropic features but instead of wire-like nanoparticles we obtained plates, faceted, pseudospherical and even some trapezoidal products that in principle do not coalesce among them to form more complex agglomerates. Other aspects indicating differences from the nanoparticles synthesized with reactive-grade heparin are the solutions tones and their UV-vis spectra, the solutions shade change from a light pink to light violet which are shown in Fig. 8.

For sample HEP1, there are three peaks: at 539, 626, and $718 \mathrm{~nm}$ and for HEP2 at 547, 642, and $737 \mathrm{~nm}$, the appearance of three peaks can be explained due to the presence of tip-pointed nanoparticles which contribute to their plasmon resonance. Additionally, the plasmon peak red-shift indicates the nanoparticles sizes are bigger.

When pharmaceutical-grade heparin concentration was changed, the nanoparticles still show anisotropic features just like when the Au content is varied, the only difference resides in the size distribution as can be observed in Fig. 9.

As the heparin concentration rises, there is more encapsulating and size-limiting effects on the particles obtained as observed from the TEM images (Fig. 9). In the case of the HEP5 sample, it is noted that some really small nanoparticles are present due the heparin reducing power (besides its stabilizing role).
The plate sizes for these three syntheses with increasing heparin concentration vary from 100 up to $800 \mathrm{~nm}$ and for the other shapes from 20 to $50 \mathrm{~nm}$.

Figure 10 shows the solutions tones as well as their UV-vis spectra. Unlike the previous case when decreasing heparin concentration case, for the three syntheses, the first peaks are located between 480 and $630 \mathrm{~nm}$, and the second one between 700 and $900 \mathrm{~nm}$, both peaks tend to blue-shift as the heparin concentration is increased indicating the nanoparticles diminish their size. Also, the FWHM for the first peak decreases from 82 to $58 \mathrm{~nm}$ indicating that the nanoparticle distribution is narrower, besides this peak sharpness indicates that a great contribution from the quasispherical nanoparticles is present. Similar mixed products have been obtained by Zhang et al. [36].

\section{Structure and changes of heparins under UV irradiation}

The two types of heparins used in nanoparticle synthesis exhibit different characteristics, as suggested by the UV-vis absorbance spectra in Fig. 11. Both present two peaks in the UV region due to the aldehyde groups present in their basic structure. For the pharmaceutical grade preparation, there is a peak related to the presence of benzyl alcohol [38] as its excipient. It should be noted that most pharmaceutical-grade

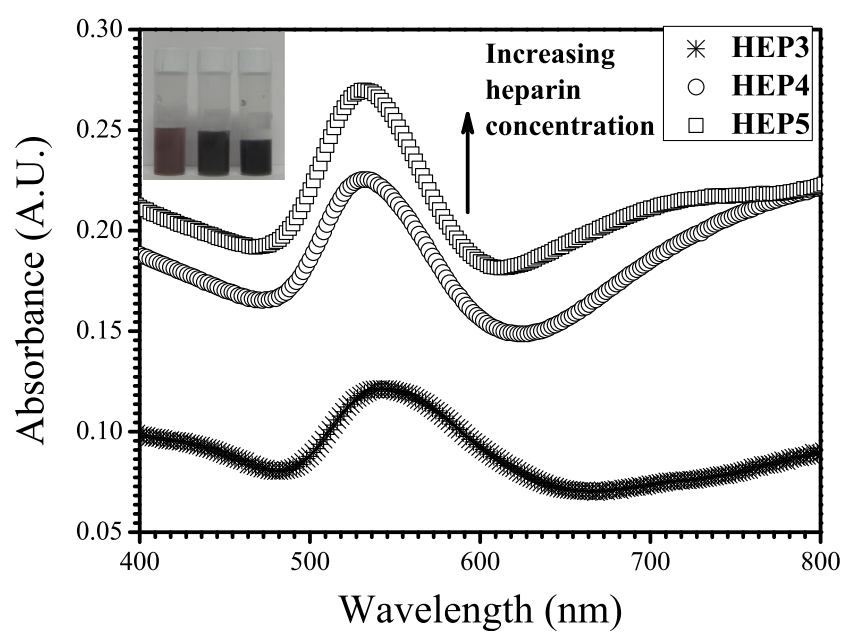

Fig. $10 \mathrm{UV}-$ vis spectra of samples synthesized with increasing heparin concentration along with the colloids prepared 

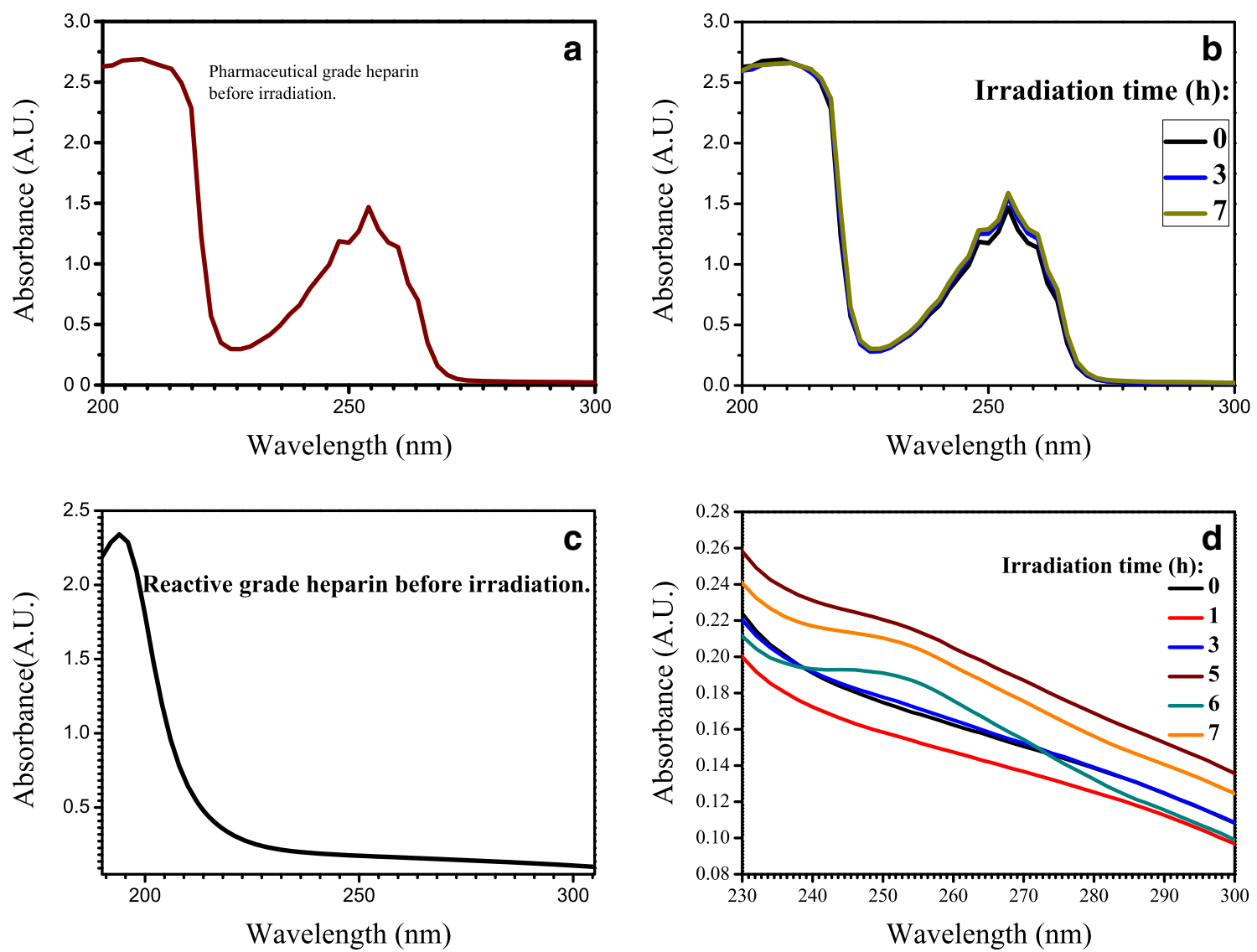

Fig. 11 UV-vis spectra of $\mathbf{a}, \mathbf{b}$ pharmaceutical and $\mathbf{c}, \mathbf{d}$ reactive grade heparin before and after UV irradiation

heparins usually contain different excipients, for example, distilled water and other substances like sodium chloride to render isotonic; sodium hydroxide and/or hydrochloric acid for $\mathrm{pH}$ adjustment, too. In our case, it was determined that pharmaceutical-grade heparin is more acidic due to benzyl alcohol presence. Balazs et al. [39] carried out several experiments using heparin, heparan sulfate and hyaluronic acid to investigate what changes occurred when exposed to UV irradiation. For heparin, they found that it is degraded by UV

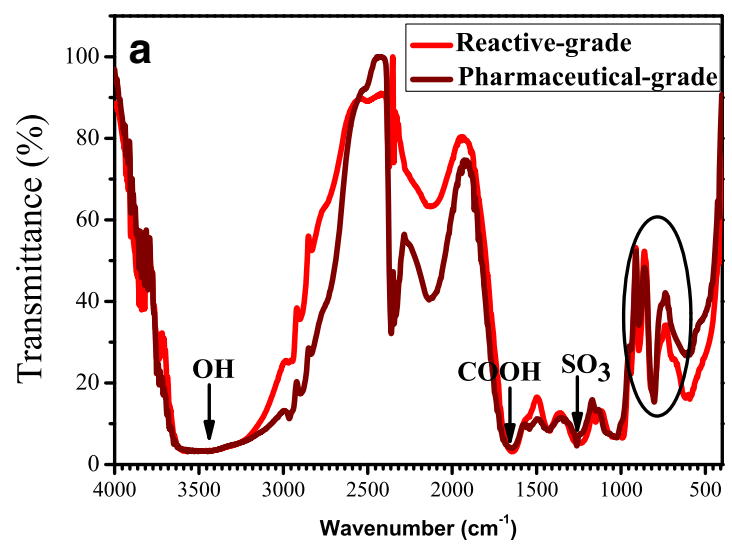

Fig. 12 FTIR spectra of heparins before they are UV-irradiated which was evident in the loss of coagulation activity because the sulfate groups are affected. It was also revealed that the heparin solutions lowered their $\mathrm{pH}$, meaning there is an electron loss. Heparin's reducing power (glucose monosaccharide) also increases proportionally to irradiation time indicating a breaking of glycosidic linkages and an increase of reducing end groups along with other irradiation products.

The spectra shown in Fig. 11a for pharmaceutical-grade heparin do not show abrupt changes in the structure, but a

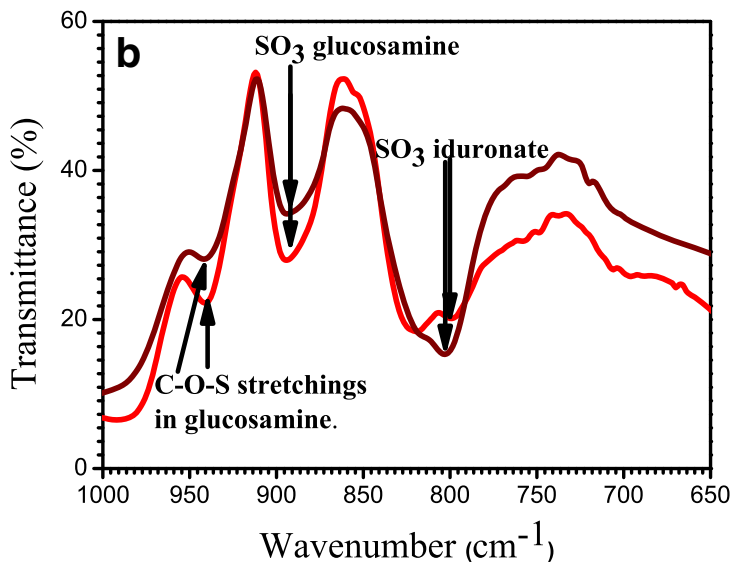



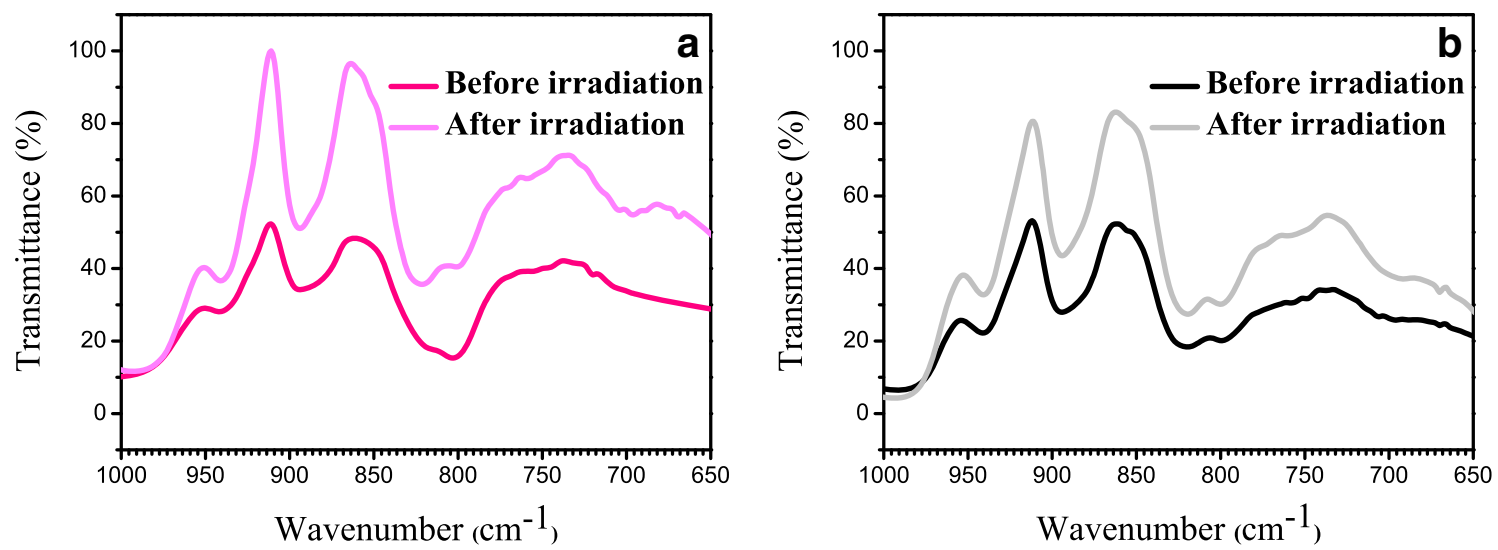

Fig. 13 FTIR spectra of a pharmaceutical-grade heparin and $\mathbf{b}$ reactive-grade heparin showing changes before and after UV irradiation

slight rise on optical density that may indicate the formation of radicals. However, for the spectra corresponding to reactivegrade heparin in Fig. 11b, the peaks are not as clear and intense as the observed for pharmaceutical heparin in Fig. 11a. This difference has to do with the different solvents in each case. Furthermore, the second peak in both cases tends to grow slightly in intensity and to change its shape as the irradiation time passes by. According to Bazals et al. [39], this may be due to the fact that the increase in absorbance is promoted in more alkaline media than in acidic media, indicating radical formation as well.

\section{FTIR peak assignation}

FTIR studies before UV irradiation taken for both heparin kinds show vibrational and absorption peaks, see Fig. 12. The spectra tend to depict some differences. One thing to take into account is the fact that heparin even when dried is still hydrophilic, which means that some peaks may correspond to solvent retention and even impurities, considering heparin synthesis yields heterogeneous products even when being from the same origin.

The peak at $3,467 \mathrm{~cm}^{-1}$ in both heparins corresponds to hydroxyl group vibrations; carboxyl and sulfate groups are responsible for the acidic character of heparin. Their location is approximately $1,645 \mathrm{~cm}^{-1}$ by $1,648 \mathrm{~cm}^{-1}$ in pharmaceutical and reactive-grade heparins, respectively. The band at approximately $1,260 \mathrm{~cm}^{-1}$ in pharmaceutical-grade heparin and the one at $1,236 \mathrm{~cm}^{-1}$ in reactive-grade heparin display different structures; these peaks and their absorption intensity are normally related to symmetric vibrations of $\mathrm{S}=\mathrm{O}$ bonds [40]. The latter has a less intense and broader peak in the 800-880 range which is the sulfated sugar region [41]. It is noted that peaks corresponding to the values of 800 and 816 and $820 \mathrm{~cm}^{-1}$ (in pharmaceutical-grade heparin and reactive-grade heparin, respectively) belong to the iduronate residue $2-0$-sulphate group and to the 6-0-sulphate group of the glucosamine residue, such bands are overlapped in pharmaceutical-grade heparin and split into two separated contributions in reactive-grade heparin but with lower absorption. Outside the mentioned range, the peak at approximately $941 \mathrm{~cm}^{-1}$ indicates vibrations of components which are part of the glucosidic linkages and also belong to $\mathrm{C}-\mathrm{O}-\mathrm{S}$ stretching (in glucosamine) [42].

Figure 13 depict the changes in both heparins after UV irradiation. The peak for the sulfate groups diminishes for pharmaceutical-grade heparin. This may indicate the appearance of other absorbing materials. The peaks that correspond to the glycosidic linkages in both heparins show absorption decrease which is in agreement with the fact that they break during irradiation as expected. The peaks corresponding to $S=$ $\mathrm{O}$ vibrations pose contrasting characteristics, because in the pharmaceutical-grade heparin there is less absorption after UV irradiation, contrary to what happens in reactive-grade heparin. This may be the result of major vibration in these bonds, from which it might be inferred that there are more atoms in this heparin chain which makes it bigger and heavier. The sulfate groups absorptions due to iduronate and glucosamine decrease in absorption but the peaks that are part of the Dglucosamine residue tend to be more defined, something that

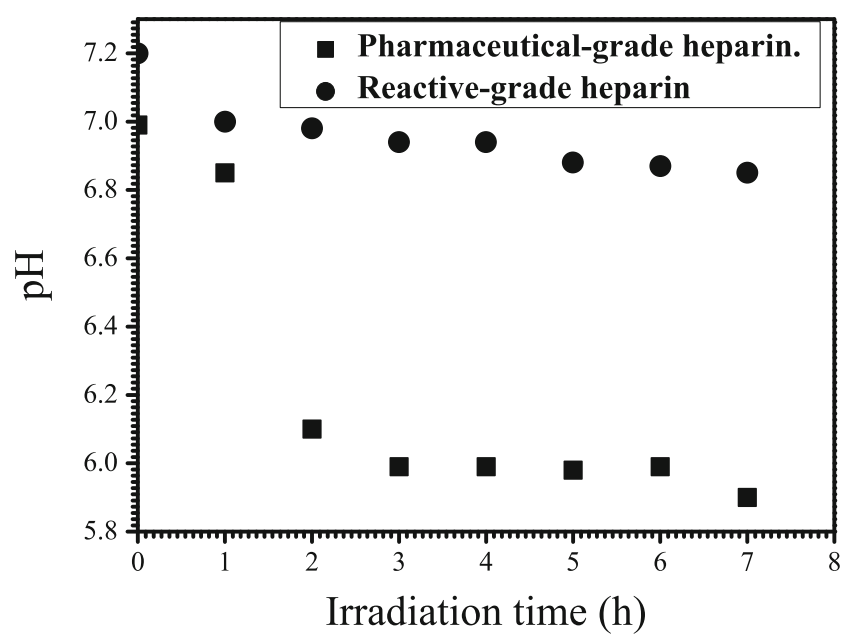

Fig. $14 \mathrm{pH}$ measurement of heparins irradiated for $7 \mathrm{~h}$ 
may be explained by suggesting an increase in the reducing end groups.

\section{Heparin $\mathrm{pH}$ measurements}

$\mathrm{pH}$ measurements were taken by preparing a reactive-grade heparin solution and a pharmaceutical grade one and irradiating them for seven hours, checking how the value changed every hour. The initial $\mathrm{pH}$ value for reactive-grade heparin was 7.2 which corresponds to an alkaline value, and from then on, it continued to go down reaching a final value of 6.85 while for the pharmaceutical-grade preparation it went from 6.99 to 5.9. That is the reason why there is more UV absorbing material generation as stated by Balazs et al. [32] and was verified in the the UV-vis spectra (Fig. 14).

Nanoparticle formation mechanism under UV irradiation

Heparin is a polysaccharide formed by disaccharide basic units (iduronic acid and glucosamine) which are repetitive along its structure with varying sulfation degrees and that becomes hydrolized when dissociated. This means that the glycosidic bond that joined them is broken because $\mathrm{H}$ and $\mathrm{OH}$ ions are donated by water. When this happens, these disaccharides units are separated (this occurs to the whole chain composed by these two monosaccharide units).

Once there has been hydrolysis, the glucosamine monosaccharide turns to its hemiacetal form. In this hemiacetal form, the aldehyde present, which $t$ is reactive in the UV range, promotes its own oxidation, which at the same time allows the reduction of gold ions when present in solution, and thus this process leads to nanoparticles generation and growth as shown in Fig. 15.

Thus, from the above discussed results, it is possible that the differences between the types of nanoparticles produced by the two heparins might be due to the fact that pharmaceuticalgrade heparin has a more acidic nature which on time allows more reduction of gold ions.

On the other hand, when working with reactive grade heparin, which is more alkaline, heavier, and bigger, the molecules might take some time for the reaction to happen in a certain direction. It may be slower, not allowing rapid
Fig. 15 Heparin-coated nanoparticle synthesis path

\section{UV irradiation.}
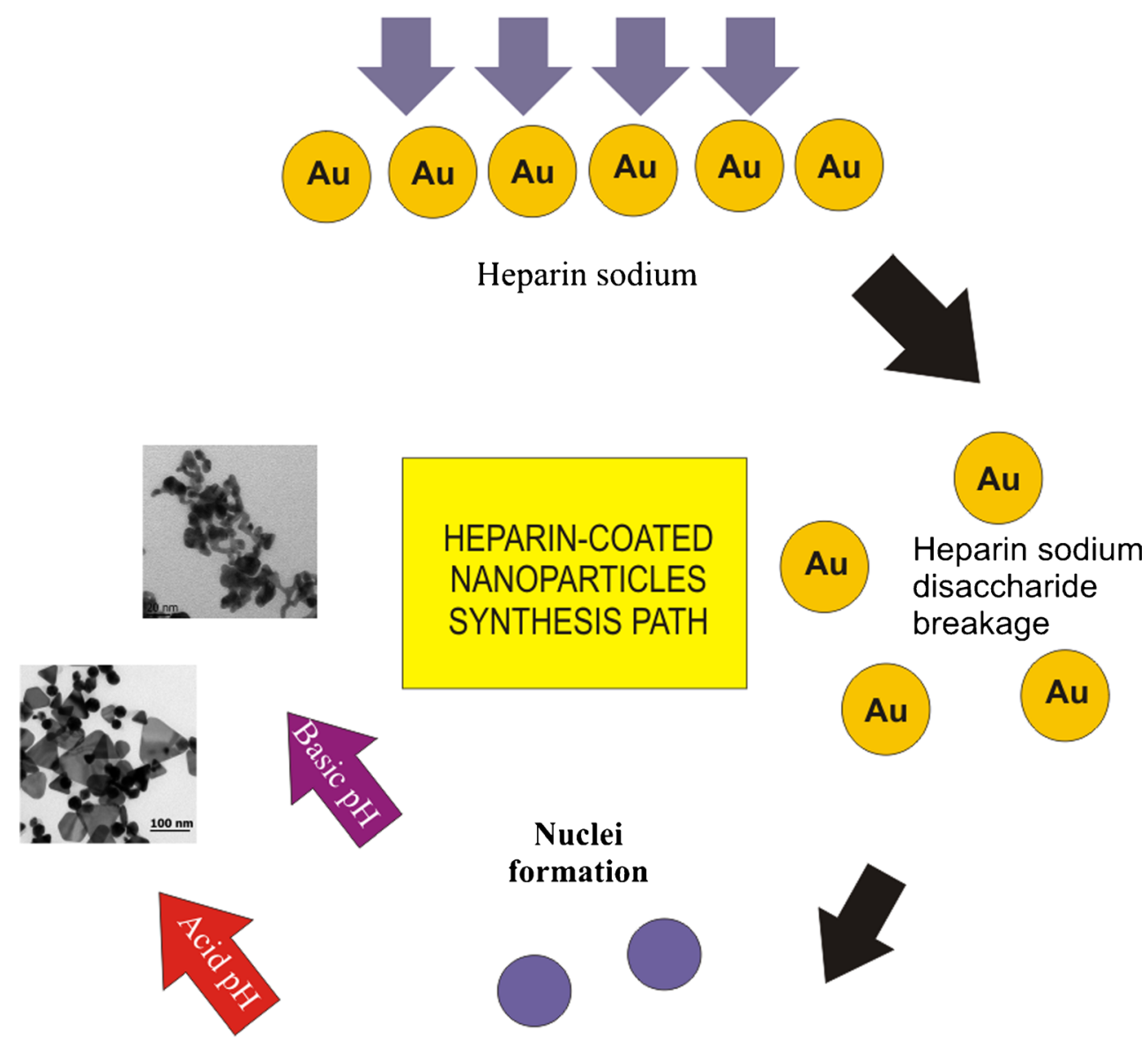

\section{Nuclei formation}
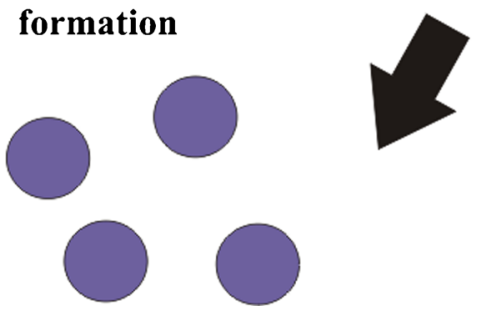
growth in other directions, and letting nanoparticles stick to each other or agglomerate instead of forming wire-like products. It has to be taken into account that polysaccharide hydrolysis is more favored in acidic than in alkaline media.

\section{Conclusions}

In summary, a simple while flexible strategy to synthesize colloidal gold anisotropic nanoparticles and nanoaggregated networks has been shown. Moreover, nanoparticles colloidal solutions are obtained by taking advantage of an inexpensive home-made black light UV reactor and heparin, a biomolecule used as an anticoagulant which is very reactive in the UV range.

It is found that low heparin concentrations trigger nanoparticle growth in preferential directions contrary to the case when its concentration is higher than the gold precursor one, which restrains this growth. It is suggested that $\mathrm{pH}$ affects the nanoparticle shapes, getting agglomerates when basic and nanoplates, faceted, pseudospherical and even some trapezoidal products when the medium is acid and agglomerated wirelike products when basic. In addition, we presume that given the biocompatibility of the capping and stabilizing agent, heparin these as-synthesized colloids could be potential candidates for plasma studies and therapy.

Acknowledgments We want to thank the following people: Ricardo Valdivia Hernandez for his technical assistance with the UV-reactor maintenance throughout this work as well as Carlos Juarez Lora, Octavio Pompa Carrera and Raul Nieto Centeno for their assistance on the UVvis spectrophotometer and FTIR system usage. TEM measurements were aided by I.B.Q. Ma. Lourdes Plama Tirado.

Open Access This article is distributed under the terms of the Creative Commons Attribution License which permits any use, distribution, and reproduction in any medium, provided the original author(s) and the source are credited.

\section{References}

1. Kimling J, Maier M, Okenve B, Kotaidis V, Ballot H, Plech A (2006) Turkevich method for gold nanoparticle synthesis revisited. J Phys Chem B 110:15700-15707. doi:10.1021/jp061667w

2. Goulet PJG, Bruce Lennox R (2010) New insights into Brust-Schiffrin metal nanoparticle synthesis. J Am Chem Soc 132:9582-9584. doi:10. 1021/ja104011b

3. Perrault SD, Chan WCW (2009) Synthesis and surface modification of highly monodispersed, spherical gold nanoparticles of 50-200 nm. J Am Chem 131:17042-17043. doi:10.1021/ja105675q

4. Kim K-S, Choi S, Cha J-H, Yeon S-H, Lee H (2006) Facile one-pot synthesis of gold nanoparticles using alcohol ionic liquids. J Mater Chem 16:1315-1317. doi:10.1039/B601478J

5. Dang TMD, Le TTT, Fribourg-Blanc E, Dang MC (2012) Influence of surfactant on the preparation of silver nanoparticles by polyol method. Adv Nat Sci: Nanosci Nanotechnol 3:035004. doi:10. 1088/2043-6262/3/3/035004, 4pp
6. Ethayaraja M, Dutta K, Muthukumaran D, Bandyopadhyaya R (2007) Nanoparticle formation in water-in-oil microemulsions: experiments, mechanism, and Monte Carlo simulation Langmuir 23: 3418-3423. doi: 10.1021/la062896c

7. Izaak TI, Babkina OV, Boronin AI, Drebushchak TN (2003) Constitution and properties of nanocomposites prepared by thermal decomposition of silver salts sorbed by Polyacrylate Matrix Colloid Journal 65:720-725. doi:10.1023/B:COLL.0000009114.41583.24

8. Yu-Ying Y, Chang S-S, Chien-Liang Lee CR, Wang C (1997) Gold nanorods: electrochemical synthesis and optical properties. J Phys Chem B Lett 101:6661-6664. doi:10.1021/jp971656q

9. Abdullah A, Annapoorni S (2005) Fluorescent silver nanoparticles via exploding wire technique PRAMANA. J Phys 65:815-819. doi: 10.1007/BF02704080

10. Richmonds C, Mohan Sankaran R (2008) Plasma-liquid electrochemistry: rapid synthesis of colloidal metal nanoparticles by microplasma reduction of aqueous cations. Appl Phys Lett 93: 131501-131504. doi:10.1063/1.2988283

11. Kim H, Heup S (2011) Moon Chemical vapor deposition of highly dispersed Pt nanoparticles on multi-walled carbon nanotubes for use as fuel-cell electrodes. Carbon 49:1491-1501. doi:10.1016/j.carbon. 2010.12.020

12. Doolittle JW Jr, Dutta PK (2006) Influence of microwave radiation on the growth of gold nanoparticles and microporous zincophosphates in a reverse micellar system. Langmuir 22: 4825-4831

13. Byrappa K, Ohara S, Adschiri T (2008) Nanoparticles synthesis using supercritical fluid technology - towards biomedical applications. Adv Drug Deliver Rev 60:299-327. doi:10.1016/j.addr.2007. 09.001

14. Chen W, Cai W, Zhang L, Wang G, Zhang L (2001) Sonochemical processes and formation of gold nanoparticles within pores of mesoporous silica. J Colloid Interf Sci 238:291-295. doi:10.1006/ jcis. 2001.7525

15. Gasaymeh SS, Radiman S, Heng LY (2010) Synthesis and characterization of silver/polyvinylpirrolidone (Ag/PVP) nanoparticles using gamma irradiation techniques. Am J Appl Sci 7(7):892-901. doi:10.3844/ajassp.2010.892.901

16. Sakamoto M, Fujistuka M, Majima T (2009) Light as a construction tool of metal nanoparticles: synthesis and mechanism. J Photochem Photobiol C Photochem Rev 10:33-56. doi:10. 1016/j.jphotochemrev.2008.11.002

17. Amendola V, Rizzi GA, Polizzi S, Meneghetti M (2005) Synthesis of gold nanoparticles by laser ablation in toluene: quenching and recovery of the surface Plasmon absorption. J Phys Chem B Lett 109(49): 23125-23128. doi:10.1021/jp055783v

18. Tan S, Erol M, Attygalle A (2007) Synthesis of positively charged silver nanoparticles via photoreduction of $\mathrm{AgNO}_{3}$ in branched polyethyleneimine/HEPES solution. Langmuir 23:9836-9843

19. Courrol LC, de Oliveira Silva FR, Gomes L (2007) A simple method to synthesize silver nanoparticles by photo-reduction. Colloid Surf A Physicochem Eng Asp 305:54-57. doi:10.1016/j.colsurfa.2007.04. 052

20. Sato-Berrú R, Redón R, Vázquez-Olmos A, Saniger JM (2009) Silver nanoparticles synthesized by direct photoreduction of metal salts. Application in surface-enhanced Raman spectroscopy. J Raman Spectroscopy 40:376-380. doi:10.1002/jrs.2135

21. Murugesan S, Xie J, Linhardt RJ (2008) Immobilization of Heparin: Approaches and Applications Current Topics in Medicinal Chem 8: 80-100. doi: 10.2174/156802608783378891

22. Wu C-C, Chen D-H (2010) Facile green synthesis of gold nanoparticles with gum arabic as a stabilizing agent and reducing agent. Gold Bull 43(4):234-240. doi:10.1007/BF03214993

23. Chien C-W, Chen D-H (2007) A facile and completely green route for synthesizing gold nanoparticles by the use of drink additives. Gold Bull 40(3):206-212. doi:10.1007/BF03215582 
24. Sivaraman SK, Kumar S, Santhanam V (2010) Room-temperature synthesis of gold nanoparticles - Size-control by slow addition. Gold Bull 43(4):275-286. doi:10.1007/BF03214997

25. Fan X, Liu L, Guo Z, Ning G, Lina Y, Zhang X (2010) Facile synthesis of networked gold nanowires based on the redox characters of aniline. Mater Lett 64:2652-2654. doi:10.1016/j.matlet.2010.08. 072

26. Castro L, Blázquez ML, Munoz JA, González F, García-Balboa C, Ballester A (2011) Biosynthesis of gold nanowires using sugar beet pulp. Process Biochem 46:1076-1082

27. Kim Y-J, Song JH (2006) Practical synthesis of Au nanowires via a simple photochemical route. Bull Korean Chem Soc 27:633-634

28. Guo Y, Yan H (2008) Preparation and characterization of heparinstabilized gold nanoparticles. J Carbohydr Chem 27:309-319. doi: 10.1080/07328300802158752

29. Park Y, Im A-R, Hong YN, Kim C-K, Kim YS (2013) Green synthesis and nanotopography of heparin-reduced gold nanoparticles with enhanced anticoagulant activity. J Nanosci Nanotechnol 11: 7570-7578. doi:10.1166/jnn.2013.6906

30. Kemp MM, Kumar A, Mousa S, Park T-J, Ajayan P, Kubotera N, Mousa S, Linhardt RJ (2009) Synthesis of gold and silver nanoparticles stabilized with glycosaminoglycans having distinctive biological activities. Biomacromolecules 10(3):589-595. doi:10. 1021/bm801266t

31. Huang H, Yang X (2004) Synthesis of polysaccharide-stabilized gold and silver nanoparticles: a green method. Carbohydr Res 339:26272631. doi:10.1016/j.carres.2004.08.005

32. Vasilev K, Zhu T, Wilms M, Gillies G, Lieberwirth I, Mittler S, Knoll W, Kreiter M (2005) Simple, one-step synthesis of gold nanowires in aqueous solution. Langmuir 21:12399-12403. doi:10.1021/ la052354f
33. Wang L, Song Y, Sun L, Guo C, Sun Y, Li Z (2008) Controllable synthesis of gold nanowires. Mater Lett 62:4124-4126. doi:10.1016/ j.matlet.2008.06.014

34. Kim Y-J, Song JH (2006) Practical Synthesis of Au Nanowires via a Simple Photochemical Route Bull Korean. Chem Soc 27(5):633-634

35. Yang S, Zhang T, Zhang L, Wang S, Yang Z, Ding B (2007) Continuous synthesis of gold nanoparticles and nanoplates with controlled size and shape under UV irradiation. Colloid Surf A Physicochem Eng 96:37-44. doi:10.1016/j.colsurfa.2006.09.017

36. Zhang G, Jasinski JB, Howell JL, Patel D, Stephens DP, Gobin AM (2012) Tunability and stability of gold nanoparticles obtained from chloroauric acid and sodium thiosulfate reaction. Nanoscale Res Lett 7:337. doi:10.1186/1556-276X-7-337

37. Lee KY, Kim M, Lee YW, Choi MY, Han SW (2007) Photosynthesis of gold nanoplates at the water/oil interface. Bull Korean Chem Soc 28(12):4124-4126

38. de Micalizzi C, Pappano NB, Debattista NB (1998) First and second order derivative spectrophotometric determination of benzyl alcohol and diclofenac in pharmaceutical forms. Talanta 47:525-530

39. Balazs EA, Laurent TC, Howe AF, Varga L (1959) Irradiation of mucopolysaccharides with ultraviolet light and electrons. Radiat Res 11:149-164. doi:10.2307/3570654

40. Sushko NI, Firsov SP, Zhbankov RG, Tsarenkov M, Marchewka M, Ratajczak C (1994) Vibrational spectra of heparins. J Appl Spectroscopy 61:5-6. doi:10.1007/BF02606421

41. Oliveira GB, Carvalho LB Jr, Silva MPC (2003) Properties of carbodiimide treated heparin. Biomaterials 24:4777-4783. doi:10. 1016/S0142-9612(03)00376-4

42. Grant D, Long WF, Moffat CF, Williamson FB (1991) Infrared spectroscopy of heparins suggests that the region $750-950 \mathrm{~cm}-'$ is sensitive to changes in iduronate residue ring conformation. Biochem J 275:193-197 